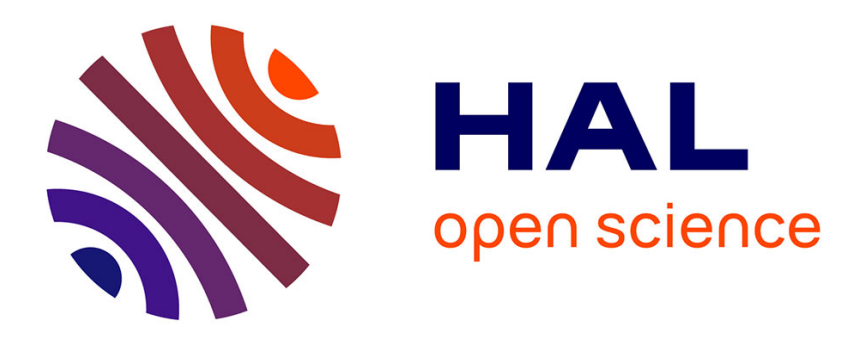

\title{
Anisotropy Of The Turbidity Of An Oriented Nematic Liquid Crystal
}

D. Langevin, M.-A. Bouchiat

\section{To cite this version:}

D. Langevin, M.-A. Bouchiat. Anisotropy Of The Turbidity Of An Oriented Nematic Liquid Crystal. Journal de Physique Colloques, 1975, 36 (C1), pp.C1-197-C1-202. 10.1051/jphyscol:1975137 . jpa00215914

\section{HAL Id: jpa-00215914 https://hal.science/jpa-00215914}

Submitted on 1 Jan 1975

HAL is a multi-disciplinary open access archive for the deposit and dissemination of scientific research documents, whether they are published or not. The documents may come from teaching and research institutions in France or abroad, or from public or private research centers.
L'archive ouverte pluridisciplinaire HAL, est destinée au dépôt et à la diffusion de documents scientifiques de niveau recherche, publiés ou non, émanant des établissements d'enseignement et de recherche français ou étrangers, des laboratoires publics ou privés. 
Classification

Physics Abstracts

$2.420-7.130$

\title{
ANISOTROPY OF THE TURBIDITY OF AN ORIENTED NEMATIC LIQUID CRYSTAL
}

\author{
D. LANGEVIN and M.-A. BOUCHIAT
}

Laboratoire de Spectroscopie Hertzienne de l'E. N. S., 24, rue Lhomond, 75231 Paris Cedex 05, France

\begin{abstract}
Résumé. - Dans un cristal liquide nématique, les fluctuations thermiques de l'orientation des molécules donnent lieu à une diffusion intense de la lumière, responsable de la turbidité caractéristique du système. Le coefficient d'absorption correspondant est égal à la section efficace totale de diffusion. Nous calculons la section efficace pour un cristal liquide orienté en tenant compte du rôle de l'anisotropie optique du milieu dans la propagation des ondes diffusées. Nous montrons que la mesure des coefficients d'absorption dans trois géométries simples permet de déterminer les trois constantes élastiques de Frank pour le même échantillon, si les indices de réfraction sont connus. Nous présentons les résultats expérimentaux obtenus avec le MBBA à température ambiante. Les valeurs mesurées des constantes élastiques sont en bon accord avec celles obtenues par d'autres procédés. Nous discutons l'influence que produit dans cette expérience la diffusion par les défauts statiques d'orientation, à l'intérieur de l'échantillon.
\end{abstract}

Abstract. - In a nematic liquid crystal, thermal fluctuations of the orientation of molecules give rise to an intense scattering of light which confers to the system a characteristic turbidity. The resulting absorption coefficient is equal to the total scattering cross-section. We compute the cross section for an oriented liquid crystal, taking into account the role of the optical anisotropy of the medium for the propagation of the scattered waves. We show that the measurement of the absorption coefficients in three simple geometries allows the determination of the three Frank elastic constants on the same sample, provided that the refractive indices are known. We give the results obtained for MBBA at room temperature. The measured values of the elastic constants are in good agreement with determinations by other methods. We also discuss the influence on this experiment coming from the scattering by static defects inside the sample.

A nematic liquid is an optically anisotropic medium, and the dielectric constant tensor depends on the orientation of the molecules. Thermal fluctuations of this orientation give rise to an intense scattering of light which was initially observed by Chatelain [1]. A theoretical interpretation of the results has been given by de Gennes [2]. This intense scattering of light confers to the system its characteristic turbidity. The resulting absorption coefficient is equal to the total scattering cross section. De Gennes [2] computed the cross section per unit solid angle within the approximation of incident and scattered light waves propagating in an isotropic medium. This approximation is to be avoided when one deals with the total cross section, since for instance it leads to an infinite cross section for certain geometries. We present here an exact computation of the cross section, starting from the recent results of Lax and Nelson [3] for light scattering in an anisotropic medium. We then show that the measurement of the absorption coefficient in three simple geometries allows the determination of the three Frank elastic constants of the nematic liquid crystal, provided that the refractive indices are known. We will give the experimental results obtained for MBBA at $23^{\circ} \mathrm{C}$. The measured values of the elastic constants are in good agreement with determinations by other methods.

Finally, we discuss the conditions for performing the measurements. One must be very careful about static orientation defects, which can produce an important forward diffraction of the incident beam and therefore must be distinguished from the scattering due to thermal fluctuations of the orientation.

1. Scattering cross section in an anisotropic medium. - A nematic liquid is optically uniaxial at each point $\mathbf{r}$, and is described by a dielectric tensor of the form :

$$
\widehat{\varepsilon}(\mathbf{r})=\varepsilon_{\perp}+\varepsilon_{\mathrm{a}}[\mathbf{v}(\mathbf{r}): \mathbf{v}(\mathbf{r})]
$$

$v(\mathbf{r})$ is the local value of the director, i. e. a unit vector parallel to the direction of the alignement of molecules at point $\mathbf{r}$.

The fluctuations $\delta \mathbf{v}$ of $\mathbf{v}(\mathbf{r})$ with respect to the mean orientation $\mathbf{v}_{0}$ (fixed by an external magnetic field, for instance) will produce fluctuations $\widehat{\delta \varepsilon}$ of $\widehat{\varepsilon}$, and will therefore scatter light. One can show [4] that the scattered electric field $\mathbf{E}_{\mathbf{d}}$ can be computed as the field 
radiated by a distribution of currents and charges in the illuminated volume :

$$
\mathbf{j}=\frac{1}{4 \pi} \frac{\partial \mathbf{P}}{\partial t} \quad \rho=-\frac{1}{4 \pi} \operatorname{div} \mathbf{P}
$$

where :

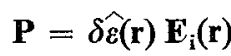

$\mathbf{E}_{\mathbf{i}}$ being the field in the medium in the absence of fluctuations. $\mathbf{E}_{\mathrm{d}}$ is solution of the following equation, derived from Maxwell's equations :

$$
\operatorname{rot} \operatorname{rot} \mathbf{E}_{\mathrm{d}}+\frac{1}{c^{2}} \frac{\partial^{2}}{\partial t^{2}}\left\{\widehat{\varepsilon}_{0} \mathbf{E}_{\mathrm{d}}\right\}=-\frac{4 \pi}{c^{2}} \frac{\partial \mathbf{j}}{\partial t}
$$

with

$$
\widehat{\varepsilon_{0}}=\varepsilon_{\perp}+\varepsilon_{\mathrm{a}} \mathbf{v}_{0}: \mathbf{v}_{0} \text {. }
$$

We will thereafter assume that $\mathbf{E}_{\mathbf{i}}$ is monochromatic, with angular frequency $\omega: \mathbf{E}_{\mathrm{i}}(\mathbf{r}, t)=\mathbf{E}_{\mathrm{i}}(\mathbf{r}) \mathrm{e}^{i \omega t}$. Neglecting the time dependence of $\hat{\delta \varepsilon}\left({ }^{1}\right)$, we then put :

$$
\mathbf{E}_{\mathrm{d}}(\mathbf{r}, t)=\mathbf{E}_{\mathrm{d}}(\mathbf{r}) \mathrm{e}^{i \omega t}
$$

Introducing the Fourier transform of $\mathbf{E}_{\mathrm{d}}(\mathbf{r})$ :

$$
\tilde{\mathbf{E}}_{\mathrm{d}}(\mathbf{k})=\int \mathbf{E}_{d}(\mathbf{r}) \mathrm{e}^{-i \mathbf{k} \cdot \mathbf{r}} \mathrm{d}_{3} r .
$$

Eq. (2) becomes :

$$
-\mathbf{k} \wedge\left[\mathbf{k} \wedge \widetilde{\mathbb{E}}_{\mathbf{d}}\right]-\frac{\omega^{2}}{c^{2}} \widehat{\varepsilon}_{0} \widetilde{\mathbf{E}}_{\mathbf{d}}=\frac{\omega^{2}}{c^{2}} \widetilde{\mathbf{P}} .
$$

For $\mathbf{P}=0$, this equation has two well known solutions : in an anisotropic medium, to every direction of propagation $\mathbf{s}=\mathbf{k} / k$, correspond two directions of the electric displacement $\boldsymbol{D}_{j}=\widehat{\varepsilon}_{0} \boldsymbol{\varepsilon}_{j}(j=1,2)$ perpendicular one to the other and to $s$, relative to the two plane waves which propagate without deformation, having a wave vector $\mathbf{k}_{j}=n_{j} \frac{\omega}{c} \mathrm{~s}$ (Fig. 1). The directions $\boldsymbol{D}_{j}$ and the refractive indices $n_{j}$ are respectively the directions and the lengths of the principal axes of the ellipse, intersection of the index ellipsoid with the plane perpendicular to $\mathbf{s}$ drawn through its center. Eq. (3) has a third solution for $\mathbf{P}=0$, which corresponds to one nonpropagating longitudinal wave with infinite refractive index $: \boldsymbol{\varepsilon}_{3} / / \mathrm{s}, 1 / n_{3}=0$ (Fig. 1).

Solving eq. (3), one finds [3-5]:

$$
\widetilde{\mathbf{E}}_{\mathrm{d}}(\mathbf{k})=\frac{\omega^{2}}{c^{2}} \sum_{j} \frac{\boldsymbol{\varepsilon}_{j}: \boldsymbol{\varepsilon}_{j}}{\frac{k^{2}}{n_{j}^{2}}-\frac{\omega^{2}}{c^{2}}} \tilde{\mathbf{P}}(\mathbf{k})
$$

where the vectors $\boldsymbol{\varepsilon}_{j}$ and $\boldsymbol{D}_{j}$ are understood to be normalized in accord with : $\boldsymbol{E}_{\boldsymbol{j}} \cdot \boldsymbol{\mathbf { O }}_{k^{\prime}}=\delta_{j k}$.

(1) The characteristic frequencies of $\delta \hat{\varepsilon}$ are much lower than $\omega$. We are only interested here in the scattered intensity integrated over all frequencies.

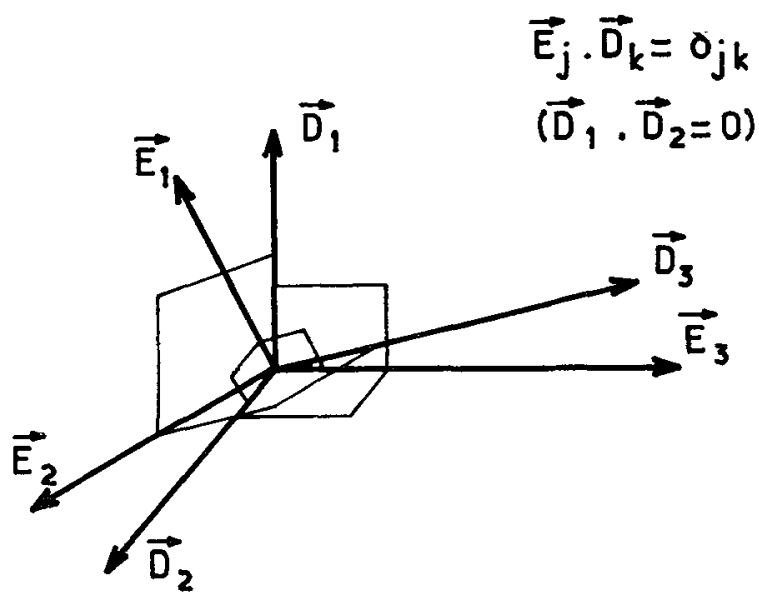

FrG. 1. - Representation of the solutions of eq. (3) for $\mathbf{P}=0$. Electric fields $\boldsymbol{\varepsilon}_{j}$ and electric displacements $\mathbf{D}_{j}=\widehat{\varepsilon_{0}} \boldsymbol{\varepsilon}_{j}$.

Taking the inverse Fourier transform of this expression, it follows that:

$$
\mathbf{E}_{\mathrm{d}}(\mathbf{r})=\int \widehat{G}\left(\mathbf{r}-\mathbf{r}^{\prime}\right) \mathbf{P}\left(\mathbf{r}^{\prime}\right) \mathrm{d}_{3} r^{\prime}
$$

where $\hat{G}(\mathbf{r})$ is the Fourier transform of the expression :

$$
\frac{\omega^{2}}{c^{2}} \sum_{j} \frac{\boldsymbol{\varepsilon}_{j}: \boldsymbol{\varepsilon}_{j}}{k^{2} / n_{j}^{2}-\omega^{2} / c^{2}}
$$

$\widehat{G}$ is the generalization of the Green function [6] for the scattered field. The interpretation of the singularities follows from physical considerations : $\mathbf{E}_{\mathrm{d}}(\mathbf{r})$ is an outgoing wave. The Fourier transform requires special care [3]. One finds :

$$
\widehat{G}(\mathbf{r})=\frac{\omega^{2}}{c^{2}} \sum_{j=1,2} n_{j}\left(\mathbf{s}_{j}\right) \boldsymbol{\xi}_{j}\left(\mathbf{s}_{j}\right): \boldsymbol{\varepsilon}_{j}\left(\mathbf{s}_{j}\right) \frac{\mathrm{e}^{i k_{j} r} \cos \delta_{j}}{4 \pi r \sqrt{K_{j}}}
$$

$\mathbf{k}_{1}$ and $\mathbf{k}_{2}$ are the wave vectors corresponding to the two waves propagating without deformation such that their Poynting vectors (the normal to the index ellipsoid) are parallel to $\mathbf{r} ; n_{j}\left(\mathbf{s}_{j}\right), \boldsymbol{\varepsilon}_{j}\left(\mathbf{s}_{j}\right)$ and $\delta_{j}$ represent the corresponding indices, vectors $\boldsymbol{E}$ and angles between $\mathbf{E}$ and $\mathbf{D}$ (Fig. 2); $K_{j}$ is the Gaussian curvature

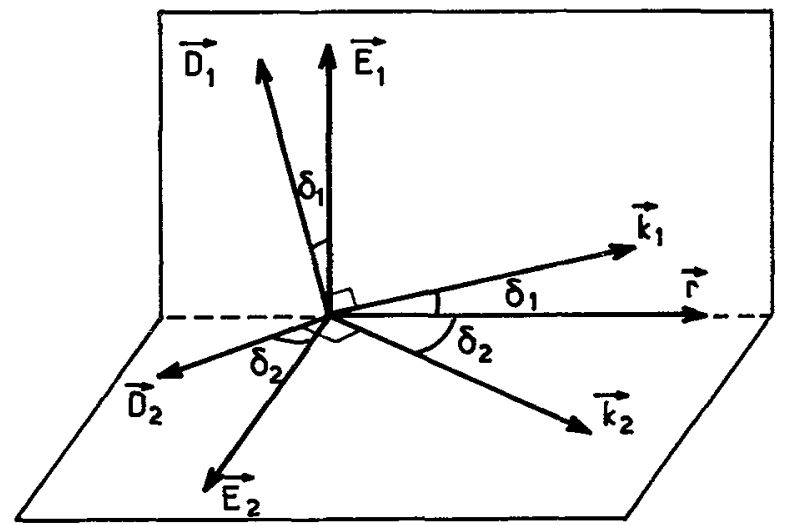

FIG. 2. - Wave vectors associated with a direction of observation $\mathbf{r}$, and the corresponding electric fields and displacements. 
of the index ellipsoid, i. e. the product of the two principal curvatures of this ellipsoid, at the point located in the direction $\mathbf{s}_{j}$.

The nonpropagating mode $(j=3)$ does not contribute to the far-field Green's dyadic.

When the incident field is of the form :

$$
\mathbf{E}_{\mathrm{i}}(\mathbf{r})=\mathrm{e}^{\mathrm{i} \mathbf{k r}} E_{\mathbf{i}} \mathbf{i}
$$

eq. (1) and (4) give the following expression for the scattered field :

$$
\mathbf{E}_{\mathrm{d}}(\mathbf{r})=\frac{\omega^{2}}{4 \pi c^{2}} \frac{E_{\mathbf{i}}}{r} \sum_{j} \frac{1}{n_{j} \cos \delta_{j} \sqrt{K_{j}}} \mathbf{f}_{j}\left[\mathbf{f}_{j} \delta \hat{\delta}\left(\mathbf{q}_{j}\right) \mathbf{i}\right] \mathrm{e}^{i \mathbf{k}, \mathbf{r}}
$$

in which $\tilde{\delta \varepsilon}\left(\mathbf{q}_{j}\right)$ is the Fourier transform of the fluctuation of the dielectric constant tensor having the wave vector $q_{j}=\mathbf{k}_{j}-\mathbf{k} ; \mathbf{f}_{j}$ is the unit vector parallel to the electric field $\boldsymbol{\delta}_{j}$.

If the incident light beam propagates in the medium along one particular mode only, characterized by the index $i$; and if $\mathrm{d} \Omega_{j}^{k}$ is the elementary solid angle relative to the wave vectors $\mathbf{k}_{j}$ around a direction $\mathbf{s}_{j}$ inside the medium $\left({ }^{2}\right)$, the cross section per unit angle and per mode $j$ of the scattered field in the direction $\mathbf{s}_{j}$ is :

$$
\begin{array}{r}
\frac{\mathrm{d} \sigma_{j}}{\mathrm{~d} \Omega_{j}^{k}}=\left(\frac{\omega^{2}}{4 \pi c^{2}}\right) \frac{1}{n_{i} \cos \delta_{i}} \frac{n_{j}}{\cos ^{2} \delta_{j}}\left[\mathbf{f}_{j} \delta \widehat{\left.\tilde{\varepsilon}\left(\mathbf{q}_{j}\right) \mathbf{i}\right]^{2} ;}\right. \\
j=1,2\left({ }^{3}\right)
\end{array}
$$

2. Total scattering cross section for an oriented nematic liquid crystal. - We turn now to the case of a nematic liquid optically uniaxial, with an optical axis parallel to the unit vector $\mathbf{v}_{0}$, having ordinary and extraordinary indices $n_{0}$ and $n_{\mathrm{e}}\left(\varepsilon_{\perp}=n_{0}^{2}, \varepsilon_{\perp}+\varepsilon_{\mathrm{a}}=n_{\mathrm{e}}^{2}\right)$. If $\theta$ denotes the angle between $\mathbf{v}_{0}$ and $\mathbf{k}$ (Fig. 3):

- $\mathbf{f}_{1}$ is in the plane of $\mathbf{k}$ and $\mathbf{v}_{0}$,

$$
1 / n_{1}^{2}=\cos ^{2} \theta / n_{0}^{2}+\sin ^{2} \theta / n_{\mathrm{e}}^{2}
$$

and

$$
\delta_{1}=\theta-\operatorname{Arctg}\left(n_{0}^{2} / n_{\mathrm{e}}^{2}\right) \operatorname{tg} \theta .
$$

This mode corresponds to the extraordinary wave;

- $\mathbf{f}_{2}$ is perpendicular to $\mathbf{k}$ and $\mathbf{v}_{0}, n_{2}=n_{0}, \delta_{2}=0$; it corresponds to the ordinary wave.

Using eq. (5) and the expression found by de Gennes for $|\mathbf{f} . \tilde{\hat{\varepsilon}}(\boldsymbol{q}) \mathbf{i}|^{2}$ in the presence of thermal fluctuations

(2) One must carefully distinguish between the solid angles relative to the direction of propagation $\mathbf{r}$ and to the wave vector $\mathbf{k}_{j}$ respectively. It can be shown [3] that : $\mathrm{d} \Omega_{j}^{k} / \mathrm{d} \Omega_{r}=\cos \delta_{j} / K_{j} n_{j}^{2}$. For the present purpose, we have found it easier to integrate $\mathrm{d} \sigma / \mathrm{d} \boldsymbol{\Omega}$ over $\mathbf{k}$ directions.

(3) Note that in the limit of an isotropic medium, $K_{j}=n_{j}^{-2}$ and $\cos \delta_{j}=1$, it can be easily verified that the expressions of the scattered field and the differential cross section are identical to the well known results in this limit [4].

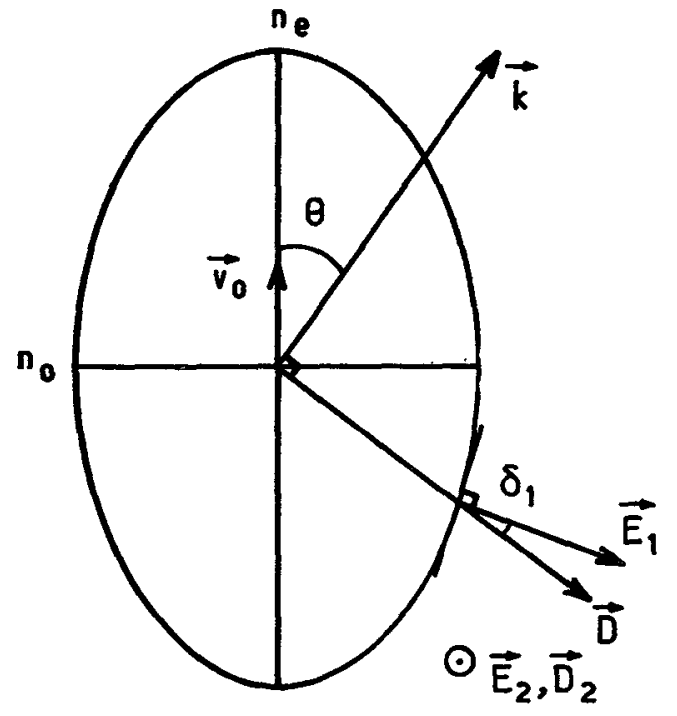

FIG. 3. - Index ellipsoid of a nematic liquid crystal. The electric fields and displacements associated with a wave vector $\mathbf{k}$.

of the orientation [2], one obtains the total scattering cross section in the form :

$$
\begin{aligned}
\sigma= & \left(\frac{\omega^{2}}{4 \pi c^{2}}\right)^{2} \frac{1}{n_{i} \cos \delta_{i}} \sum_{j=1,2} \int \mathrm{d} \Omega_{j}^{k} \frac{n_{j}}{\cos ^{2} \delta_{j}} \times \\
& \times \sum_{\alpha=1,2} \varepsilon_{\mathrm{a}}^{2} k T \frac{\left[\left(\mathbf{e}_{\alpha} \cdot \mathbf{i}\right)\left(\mathbf{f}_{j} \cdot \mathbf{v}_{0}\right)+\left(\mathbf{e}_{\alpha} \cdot \mathbf{f}_{j}\right)\left(\mathbf{i} \cdot \mathbf{v}_{0}\right)\right]^{2}}{K_{33} q_{z_{j}}^{2}+K_{\alpha \alpha} q_{\perp_{j}}^{2}+\chi_{\mathrm{a}} H^{2}}
\end{aligned}
$$

where $e_{2}$ is a unit vector normal to $\mathbf{v}_{0}$ and to $\mathbf{q}$, $\mathbf{e}_{1}=\mathbf{v}_{0} \wedge \mathbf{e}_{2} \cdot q_{z}=\mathbf{q} \cdot \mathbf{v}_{0}, q_{\perp}=\mathbf{q} \cdot \mathbf{e}_{1} ; \chi_{\mathrm{a}}$ is the anisotropy of the magnetic susceptibility and $H$ the magnetic field ; $K_{11}, K_{22}$ and $K_{33}$ are the Frank elastic constants.

We have computed the total scattering cross section in three geometries : (1) $\mathbf{k} / / \mathbf{v}_{0}$; (2) $\mathbf{k} \perp \mathbf{v}_{0}, \mathbf{i} \perp \mathbf{v}_{0}$; (3) $\mathbf{k} \perp \mathbf{v}_{0}, \mathbf{i} / / \mathbf{v}_{0}$ [5]. For typical magnetic fields (less, than $10 \mathrm{kG})$, the contribution of the term $\chi_{\mathrm{a}} H^{2}$ in eq. (6) is found to be negligible $\left({ }^{4}\right)$.

We give in the appendix the results obtained as integrals of the variable $u=\cos \theta$, with the reduced quantities :

$$
\begin{gathered}
a_{\alpha}=\frac{K_{\alpha \alpha}}{K_{33}}, \quad(\alpha=1,2) ; \quad b=\frac{n_{\mathrm{e}}}{n_{0}} ; \\
\sigma_{0}=\frac{k T}{4 \pi} \frac{\omega^{2}}{c^{2}} \frac{\varepsilon_{\mathrm{a}}^{2}}{K_{33} n_{0}^{2}} .
\end{gathered}
$$

The values of $\sigma / \sigma_{0}$ have been derived numerically with a computer for different values of the parameters $a_{\alpha}$ and $b$. We found important differences between the three geometries: this anisotropy of $\sigma$ is due to the anisotropy of the elasticity $\left(a_{\alpha} \neq 1\right)$ and to the optical anisotropy $(b \neq 1)$. If $b$ and $\varepsilon_{\mathrm{a}}=n_{\mathrm{e}}^{2}-n_{0}^{2}$ are given,

(4) For zero magnetic field, the assumption of an optically isotropic medium leads to an infinite cross section in geometries 2 and 3. 
i. e. if the refractive indices are known, the measurement of $\sigma$ in the three geometries allows the determination of the three elastic constants.

3. Experimental results. - We have measured the absorption coefficient using a He-Ne laser of $50 \mathrm{~mW}$ on which was mounted a rotator of polarization allowing modifications of the polarization of the incident beam without any geometric displacement. A permanent magnet was used to orient the liquid contained in a sealed pyrex cell. The solid angle of detection was varied by means of a diaphragm and the transmitted light beam was detected by a photocell. In geometry 1 the value of the magnetic field was $3000 \mathrm{G}$ and in geometries 2 and $3,1000 \mathrm{G}$. The cells were cylindrical, their thickness e varying between 1 and $2 \mathrm{~mm}$.

We found in certain geometries that the transmitted intensity was strongly dependent on the aperture of the cone used as solid angle of detection, measured by the angle $2 \psi$. The results obtained at $23^{\circ} \mathrm{C}$ in a very pure sample (clearing point of $45.5^{\circ} \mathrm{C}$ ) $1.3 \mathrm{~mm}$ thick, are plotted in figure 4 for the three geometries under consideration as a function of the angle $\psi$. Such a large variation, particularly in geometry 3 , for values of $\psi$ ranging between 0.1 and $0.5^{\circ}$, was at first unexpected since expressions (5) and (6) predict that the fraction of light intensity scattered forward by the fluctuations

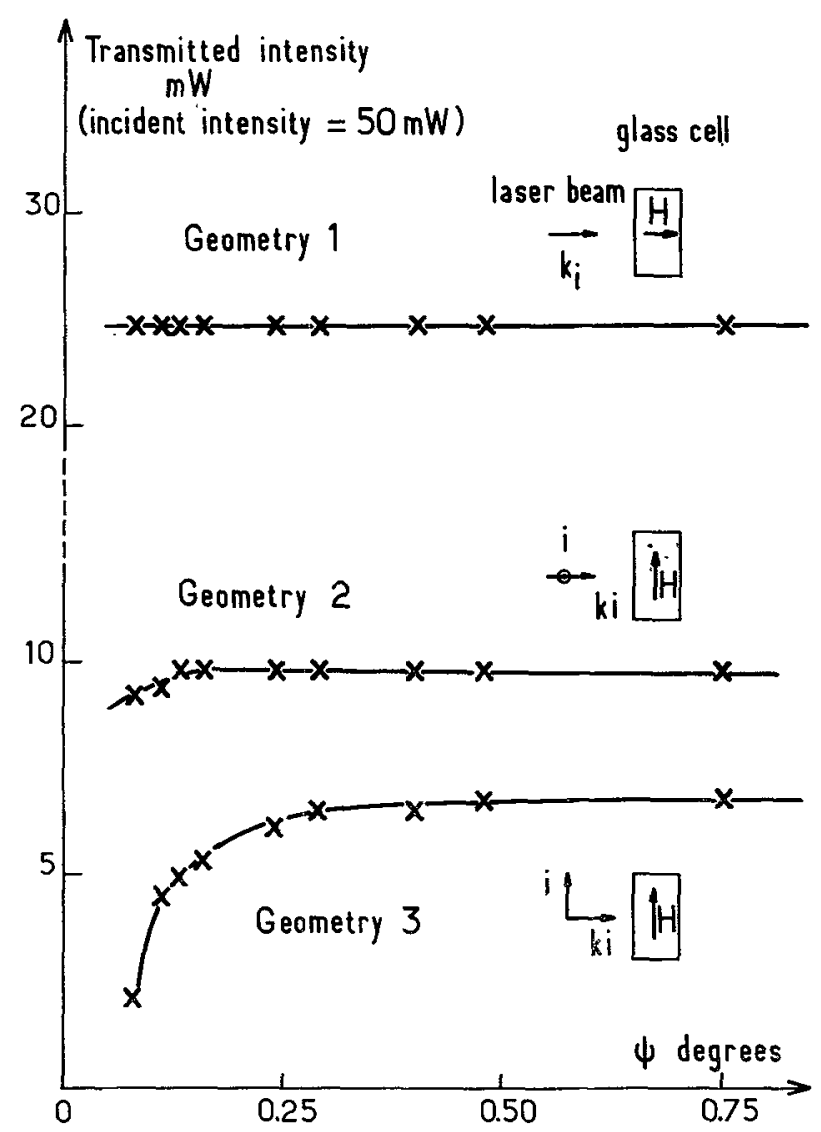

Frg, 4. - Transmitted intensities by a MBBA cell $1.3 \mathrm{~mm}$ thick at $23^{\circ} \mathrm{C}$, as a function of the half aperture $\psi$ of the cone used as solid angle of detection. inside such a small solid angle is negligible. One also notes that the angle $\psi$, though small, is much larger than the angular divergence of the incident laser beam (roughly $0.02^{\circ}$ ).

We interpreted this behaviour as due to the scattering caused by static orientation defects in the sample. The scattering cross section relative to the defects $\sigma^{\mathrm{d}}$ is still given by formula (6) providing $k T$ is replaced by $F(q)$, the defect energy. If one admits that these defects are created by the planeity defects of the glass surface, the wave vectors involved are very small. This means that $F(q)$ increases rapidly when $q$ goes to zero. As a result, the intensity scattered by the defects is strongly concentrated around the transmitted beam. One can see that $\sigma_{2}^{\mathbf{d}}$ is negligible compared to $\sigma_{3}^{\mathrm{d}}$ because, for small studied scattering angles, it involves much larger wave vectors : $q \gtrsim\left(n_{\mathrm{e}}-n_{0}\right) \omega / c$, the contribution to $\sigma_{2}^{\mathrm{d}}$ of the polarization $\mathbf{f}$ parallel to $i$ being zero. With the same kind of reasoning one would expect $\sigma_{1}^{\mathrm{d}}$ and $\sigma_{3}^{\mathrm{d}}$ to be of the same order of magnitude, but in fact they involve different types of defects and different energies $F(q)$. By measuring the reflectivity of the nematic-glass interfaces, we found that the molecules are normal to the surfaces of the glass cell $\left({ }^{5}\right)$. One can then expect less defects in the cell in geometry 1 where the magnetic field is parallel to the molecules close to the glass surfaces. In geometries 2 and 3 , there are two distorded regions in the vicinity of the glass surfaces, of thickness $\xi \sim \sqrt{K_{33} / \chi_{\mathrm{a}}} H^{-1}$. We can reasonably assume that there is a cut-off value for $F(q)$ which is $q^{*} \sim 1 / \xi$. Then if we use a solid angle of detection $\psi>q^{*} / k_{i}$, we detect not only the transmitted light but also all the light scattered forward by the defects. In such conditions, the detected intensity becomes practically constant in a wide range of $\psi$, as expected, and can be interpreted as all the light which is not scattered by thermal fluctuations. This condition :

$$
\psi>\frac{1}{k_{i}} \sqrt{\frac{\chi_{\mathrm{a}}}{K_{33}}} H \sim 0.2^{\circ}
$$

explains satisfactorily the behaviour of the experimental curves presented on figure 4 .

We have plotted in figure 5 the results obtained in geometry 3 for different sample thicknesses $e$. The transmitted intensities present the same characteristical behaviour for $\psi \lesssim 0.2^{\circ}$. This is in agreement with our interpretation. For $e=2 \mathrm{~mm}$, the transmitted intensity never reaches a limiting value: for such values of $e$, the scattered intensity becomes quite large. The fraction of this intensity collected in the solid angle of detection is probably of the same order of magnitude as the transmitted intensity. Therefore, we limited ourselves to the interpretation of the results obtained for smaller thicknesses. By taking the limit values of

(5) Before filling the cell, we cleaned it carefully by heating in a flame. This is known to favour homeotropic configurations. 


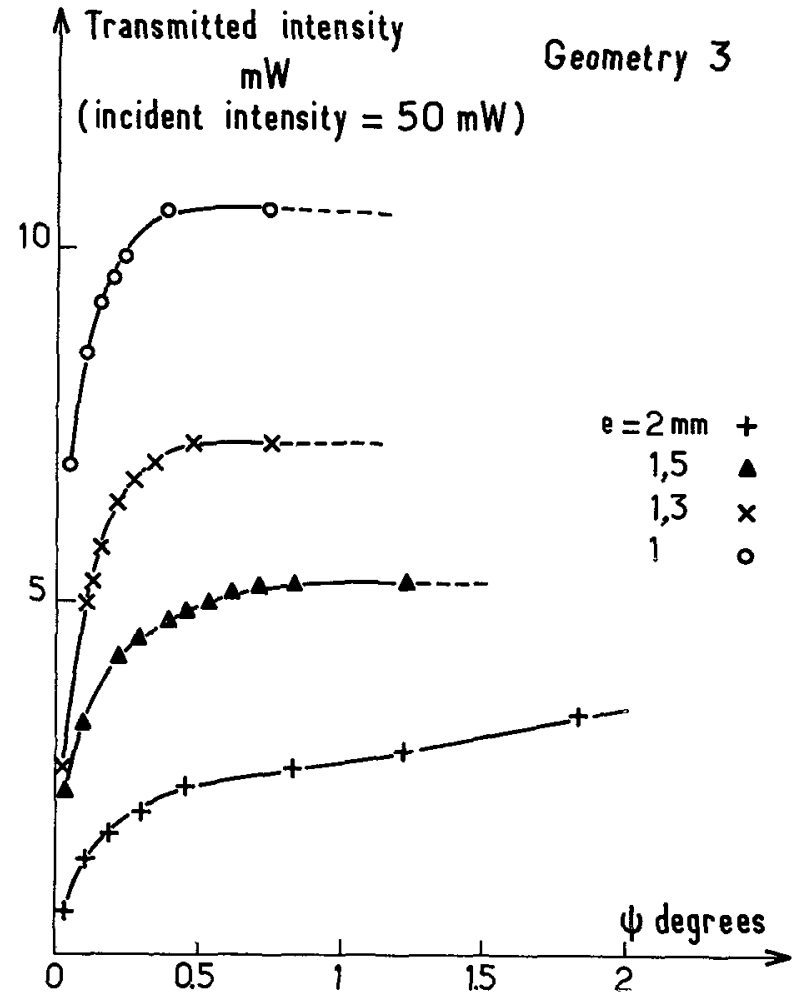

FIG. 5. - Transmitted intensities by MBBA cells of different thicknesses at $23^{\circ} \mathrm{C}$, as a function of $\psi$.

the transmitted intensities, we obtain the following values for the absorption coefficients :

\begin{tabular}{|c|c|c|c|}
\hline$e \mathrm{~mm}$ & $\sigma_{1}$ & $\sigma_{2}$ & $\sigma_{3}$ \\
\hline 1 & $4.9 \mathrm{~cm}^{-1}$ & $12.1 \mathrm{~cm}^{-1}$ & $14.9 \mathrm{~cm}^{-1}$ \\
\hline 1.3 & 4.9 & 12.1 & 14.7 \\
\hline 1.5 & 4.9 & 12.1 & 14.8 \\
\hline 2 & 5 & & \\
\hline
\end{tabular}

Using the values of the refractive indices measured by Haller [7] we deduce :

$K_{11}=6.7 \times 10^{-7} \mathrm{dyne} / \mathrm{cm}, K_{22}=4.2 \times 10^{-7} \mathrm{dyne} / \mathrm{cm}$ and

$$
K_{33}=8.4 \times 10^{-7} \text { dyne } / \mathrm{cm} .
$$

There are several uncertainties in these values:

- The uncertainty of the refractive indices which is estimated to be about $1.5 \%$ [7] and leads to an uncertainty for the elastic constants which is twice as large. This comes from the fact that the absorption anisotropy depends more on the birefringence than on the anisotropy of elasticity.

- The effect of the regions in the vicinity of the glass surfaces which are not oriented by the magnetic field in geometries 2 and 3 . We have estimated the order of magnitude of the error on $\sigma_{i}$ by the expression : $2 \xi\left(\sigma_{i}-\sigma_{1}\right) / e$, which is approximatively $3 \%$. This effect is obviously unfavourable for very thin samples.

- The other possible absorption phenomena. The corresponding uncertainty is of the order of the absorption coefficient of the isotropic phase $\sigma \sim 0.2 \mathrm{~cm}^{-1}$, that is approximatively $2 \%$.

The values of the elastic constants obtained are therefore uncertain to approximatively $10 \%$.

The measured values are in a fairly good agreement with those reported in the literature [8].

This new method for measuring elastic constants allows the determination of the three constants for the same sample in a very easy way. However the role played by the defects must be correctly taken into account.

We now plan to apply this method to a second-order nematic to smectic A transition where the elastic constants $K_{22}$ and $K_{33}$ diverge, and consequently must considerably affect the turbidity anisotropy of the system.

Acknowledgments. - We wish to thank F. Rondelez for-very helpful discussions.

\section{References}

[1] Chatelain, P., Acta Cristallogr. 4 (1951) 453.

[2] De Gennes, P. G., C. R. Hebd. Séan. Acad. Sci. 266B (1968) 15.

[3] LAX, M. and NeLson, D. F., in Proceedings of the Third Rochester Conference on Coherence and Quantum Optics, edited by L. Mandel and E. Wolf (Plenum Press, New York) 1973 (eq. (2-1), (2-11), (2-15), (2-16) and (4-12)), and Phys. Rev. B 4 (1974) 3694, XII, Appendix $F$.

[4] FabelinSKII, J. L., Molecular Scattering of Light (Plenum Press) 1968, chap. 1.
[5] Langevin, D., Thesis, Paris, 1974.

[6] JACKson, J. D., Classical Electrodynamics (Ed. Wiley) 1963, chap. 6.

[7] Haller, I., Huggins, H. A. and Freiser, M. J., Mol. Cryst. Liqu. Cryst. 16 (1972) 53.

[8] See for instance, Rondelez, F. and Hulin, J. P., Solid State Commun. 10 (1972) 1009 ;

Williams, C. and Cladis, P. E., Solid State Commun. 10 (1972) 357

LÉGER, L., Solid State Commun. 10 (1972) 697 ;

HALIER, I., J. Chem. Phys. 57 (1972) 1400.

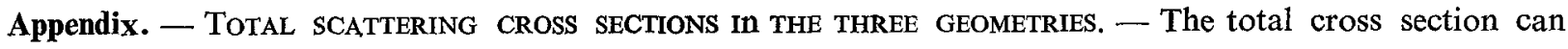
be expressed in the following form [5]:

Geometry $1, \mathbf{k} / / \mathbf{v}_{0}$

$$
\frac{\sigma_{1}}{\sigma_{0}}=\frac{I_{0}\left(a_{1}\right)+I_{0}\left(a_{2}\right)}{4}
$$


Geometry $2, \mathbf{k} \perp \mathbf{v}_{0}, \mathbf{i} \perp \mathbf{v}_{0}$

$$
\frac{\sigma_{2}}{\sigma_{0}}=I_{1}\left(a_{2}\right)+I_{2}\left(a_{1}\right)-I_{2}\left(a_{2}\right)
$$

Geometry $3, \mathbf{k} \perp \mathbf{v}_{0}, \mathbf{i} / / \mathbf{v}_{0}$

$$
\frac{\sigma_{3}}{\sigma_{0}}=I_{3}\left(a_{2}\right)+I_{4}\left(a_{1}\right)-I_{4}\left(a_{2}\right)+I_{5}\left(a_{1}\right)+I_{6}\left(a_{2}\right)-I_{6}\left(a_{1}\right)
$$

with

$$
\begin{aligned}
I_{0}(a)= & \int_{-1}^{+1} \mathrm{~d} u \frac{b\left(1-u^{2}\right)\left[1+\left(b^{2}-1\right) u^{2}\right]^{-3 / 2}}{\left[b u-\sqrt{\left.1+\left(b^{2}-1\right) u^{2}\right]+a b^{2}\left(1-u^{2}\right)}\right.} \\
I_{1}(a)= & \int_{0}^{1} \mathrm{~d} u \frac{b\left(1-u^{2}\right)\left[1+\left(b^{2}-1\right) u^{2}\right]^{-3 / 2}}{\sqrt{\left[u^{2}\left(b^{2}-a\right)+a\left(1+b^{2}\right)\right]^{2}-4 a^{2} b^{2}\left[1+\left(b^{2}-1\right) u^{2}\right]\left(1-u^{2}\right)}} \\
I_{2}(a)= & \frac{1}{4 a b} \int_{0}^{1} \frac{\mathrm{d} u}{u^{2}} \frac{1-u^{2}}{\left[1+\left(b^{2}-1\right) u^{2}\right]^{5 / 2}} \times \\
& \times\left\{\sqrt{\left[u^{2}\left(b^{2}-a\right)+a\left(1+b^{2}\right)\right]^{2}-4 a^{2} b^{2}\left[1+\left[b^{2}-1\right] u^{2}\right]\left[1-u^{2}\right]}-b^{2} u^{2}-a\left|1-b^{2}+\left(2 b^{2}-1\right) u^{2}\right|\right\} \\
I_{3}(a)= & \frac{1}{b} \int_{0}^{1} \frac{\mathrm{d} u}{\sqrt{\left[u^{2}(1-a)+a\left(1+b^{2}\right)\right]^{2}-4 a^{2} b^{2}\left(1-u^{2}\right)}} \\
I_{4}(a)= & \frac{1}{4 a b} \int_{0}^{1} \frac{\mathrm{d} u}{u^{2}\left(1-u^{2}\right)}\left\{\sqrt{\left[u^{2}(1-a)+a\left(1+b^{2}\right)\right]^{2}-4 a^{2} b^{2}\left(1-u^{2}\right)}-\left[u^{2}(1+a)+a\left(b^{2}-1\right)\right]\right\} \\
I_{5}(a)= & \int_{0}^{1} \mathrm{~d} u \frac{b^{2} u^{2}\left[1+\left(b^{2}-1\right) u^{2}\right]^{-3 / 2}}{\sqrt{\left[u\left(1+a b^{2}-2 a\right)+2 a\right]^{2}-4 a^{2}\left(1-u^{2}\right)\left[1+\left(b^{2}-1\right) u^{2}\right]}} \\
I_{6}(a)= & \frac{b^{2}}{4 a} \int_{0}^{1} \frac{\mathrm{d} u}{\left(1-u^{2}\right)}\left[1+\left(b^{2}-1\right) u^{2}\right]^{-3 / 2} \times \\
& \times\left\{\sqrt{\left[u^{2}\left(1+a b^{2}-2 a\right)+2 a\right]^{2}-4 a^{2}\left(1-u^{2}\right)\left[1+\left(b^{2}-1\right) u^{2}\right]}-u^{2}\left(1+a b^{2}\right)\right\} .
\end{aligned}
$$

